\title{
Automatic Generation of CFD Mesh for Mountainous Regions
}

\author{
Huibo Dang ${ }^{1, a}$, Hongjie Zhang ${ }^{2, b}$ and Huixue Dang ${ }^{3, c, *}$ \\ ${ }^{1}$ School of Computer Engineering, Xi'an Aeronautical Polytechnical Institute, Xi’an, Shaan’xi, China \\ ${ }^{2}$ China Electric Power Research Institute, Beijing, China \\ ${ }^{2}$ School of Civil Engineering, Chang'an University, Xi'an, Shaan’xi, China \\ a zhoulinshijie@126.com, ${ }^{\mathrm{b}}$ zhanghongjie1@epri.sgcc.com.cn, ${ }^{\mathrm{c}}$ dhxlxz@126.com \\ *corresponding author
}

Keywords: micro-terrain of mountainous region, mesh; automatic generation, continuous and smooth connection, domain interface.

\begin{abstract}
Wind resistant design of ultra-high voltage transmission lines is greatly influenced by wind flow characteristics of complex terrains in mountainous regions. To obtain wind flow files over undulating hills with complex shapes and different elevations, an automatic mesh generation software is designed and developed. First, the elevation data is obtained from Aster GDEM V2 of NASA. Then geometric surfaces are generated by using continuous and smooth connection technique. Next, accounting for the demands of different wind directions, the CFD model is designed to be consisted of inner rotational region and outer fixed region, and flow data is exchanged at these two domain interfaces. Then, mixed prism/tetrahedron mesh is generated for inner rotational region. Last, the height parameters at the interface boundary of inner rotational region is copied to the outer fixed region, and fully hexahedral mesh is generated. The generated meshes of these two different regions are written in the ".cas" data format of fluent software, respectively, hence generality of data format is guaranteed. By checking the grids by using fluent software, it is showed that, the automatically generated mesh satisfies the demands for CFD simulations.
\end{abstract}

\section{Introduction}

Electric power is transferred from western region by passing through wide mountain regions with complex topography by ultra-high voltage transmission lines. Due to the heat transfer as well as topography variation in mountainous regions, atmospheric wind flow is greatly changed when it flow upon undulating hills and hilly areas ${ }^{[1]}$. Thus, local flow characteristics will be evidently different from the result captured by meteorological station. Hence, the buildings and constructions designed based on the standards and codes of plat ground may be destructed by local strong winds ${ }^{[2]}$. However, the standards and codes commonly used rarely describe the local flow induced by mountain regions. Meanwhile, the wind flow characteristics induced by high elevation, high temperature variation, and deep-cutting gorge are rarely investigated.

In the published literatures, Daniel S. Abdi ${ }^{[3]}$ et al. pointed out that, real topography contain irregular three dimensional topographic features that are surrounded by other topographic features, and such difference often leads to overly conservative design of structures located on complex terrain. The problem is pronounced for long span structures such as transmission lines that cross multiple zones with different speed-up and turbulence characteristics, especially at the crest of hills and escarpments, where the fractional speed up ratio can be highly enough to cause major structural failures. Hence, many national standards and codes, such as NBCC, ASCE-7, AS/NZS 1170.2, EUROCODE 1, etc., provide genal guidelines for estimating topographical multiplication factors of wind speed-up over hill crests and escarpments. However, the guidelines presented in the above 
codes are for simple hilly geometries. While for complex mountain zones, the codes suggested wind tunnel tests and CFD simulations.

As wind-sensitive large-scale structures, one of the problem in the study of transmission lines across mountainous zones in their designing and evaluation process is that, manual mesh generation usually cost great human labour and working time ${ }^{[4]}$. Thus, automatic mesh generation is of nice choice and several researchers have attributed their work on this key point.

In the automatic generation of mesh over complex geometries, unstructured tetrahedron mesh is widely employed, and tetrahedrons and prisms are usually adopted together to discretize computational zones. Comparing to triangles and tetrahedrons, quadrilaterals and hexagons can discretize computational zones with less elements but high computational fidelity, and they are also distortion-insensitive, hence, they are usually of first choice in grid generation on surface and solid geometry models. However, due to the defect of topology division of quadrilaterals and hexagons, automatic mesh generation by using these elements are not fully resolved. But unstructured tetrahedron mesh generation is well developed, such as Muller ${ }^{[5]}$ et al. combined advancing front method and Delaunay method together for unstructured surface mesh generation. Frey ${ }^{[6]}$ developed it to be three dimensional and it was found to be with both the high quality characteristic of advancing front method and the high efficiency and convergence characteristics of Delaunay method.

WU Huo-zhen ${ }^{[7]}$ presented a 3D constrained Delaunay triangulation algorithm based on the classical 3D Delaunay tetrahedral mesh generation method, and the 3D finite element mesh for complex region under different geological conditions could be automatically generated in the civil engineering; Also, the high quality of the mesh which could be guaranteed provides sufficient condition for the high-precision finite element calculation. Jonathan Richard Shewchuk ${ }^{[8]}$ pointed out that, constrained Delaunay tetrahedralizations maintain most of the favorable properties of ordinary Delaunay tetrahedralizations, but some sets of constraining segments and facets simply do not have constrained Delaunay tetrahedralizations. This problem is fixed by and boundary conformity can always be enforced by judicious insertion of additional vertices, combined with constrained Delaunay tetrahedralizations. Daming Feng ${ }^{[9]}$ et al. presented a scalable three dimensional hybrid parallel Delaunay image-to-mesh conversion algorithm (PDR.PODM) for distributed shared memory architectures. David Eller ${ }^{[10]}$ combines the Delaunay-based tetrahedral mesh generator TETGEN and a novel technique for creation of a prismatic layer together, thus an open source implementation of an efficient mesh generation procedure for hybrid prismatictetrahedral meshes intended for use in Reynolds-averaged Navier-Stokes solutions is developed.

The published progress in unstructured mesh generation can be nice reference in automatic grid generation, however, for transmission lines cross different mountainous zones, these work above can not be employed directly. In order to obtain wind flow field around transmission lines fast and accurately, automatic mesh generation will be discussed in present study.

\section{Geometry surface of complex terrains in mountainous regions}

Geometry surface of complex terrains in mountainous regions is usually obtained from highfidelity maps, geographic information framework data, etc.. Among different digital elevation models(DEM), such as ASTER GDEM, SRTM, and GTOPO30 etc., ASTER GDEM covers the most wide land surfaces (between 83N and 83S, comprised of $227021^{\circ} \times 1^{\circ}$ tiles) with high resolution( per 1 second), thus it is selected for geometry surface generation. By adopting the opensource dynamic library LibGeoTIFF, the elevation data is achieved, and then noise points are deleted. An example of geometry model is shown in Fig.1. 


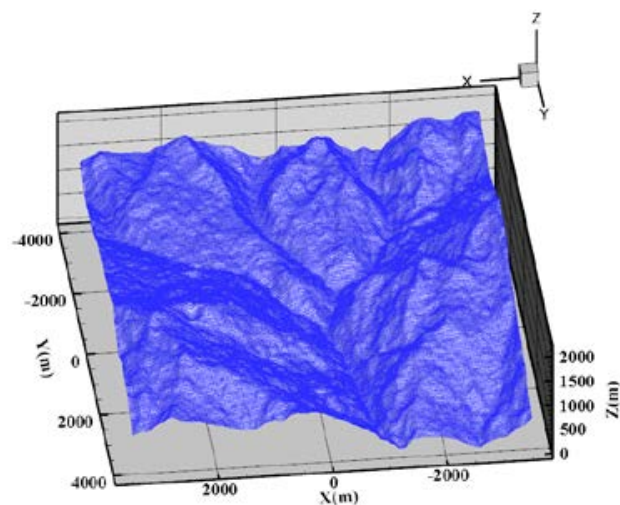

(a) point cloud surface

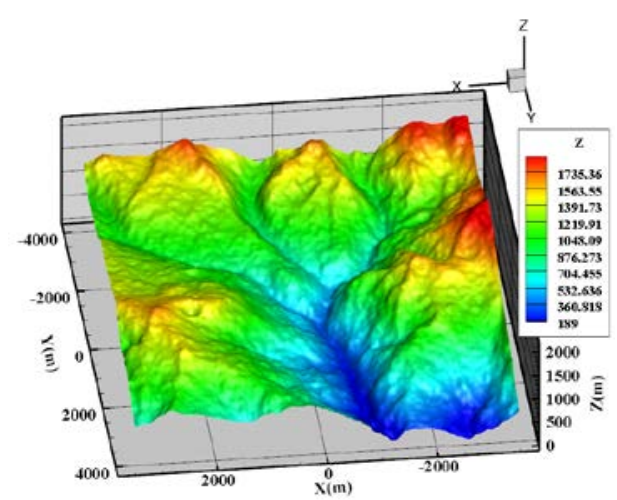

(b) elevation of the surface

Fig.1 Elevation contours of geometry model from ASTER GDEM points

\section{Automatic mesh generation}

\section{Division of computational zones and boundary conditions}

In both CFD simulations and wind tunnel tests of micro terrain wind flows, the effect of wind incidence angle on flow characteristics should be accounted for. In order to simulate wind incidence angle, by referring to the rotational gauge in test section of wind tunnel, the computational domain is divided into two parts: a) inner rotational domain and b) outer zone, as shown in Fig.2. Flow data exchange inbetween these two domains is achieved by using interface boundary conditions, where mass conservation, momentum conservation are both satisfied.

The boundary conditions of inner rotational zone are wall for up boundary, micro-terrain, and interface for the cylinder; the boundary conditions for outer domain are velocity inlet for atmospheric wind profile, outflow boundary such as pressure outlet, free-slip wall boundary for up wall, symmetry boundary, and wall boundary for bottom wall. These boundary conditions are plotted as shown in Fig.3.

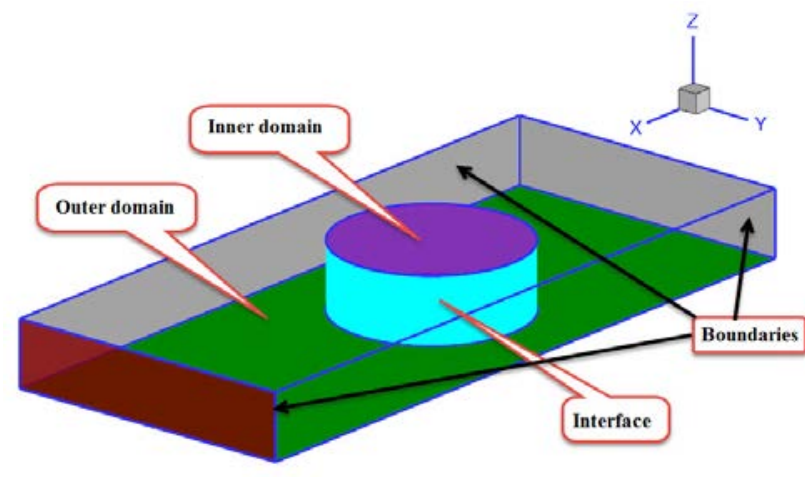

Fig.2 Domains and boundaries

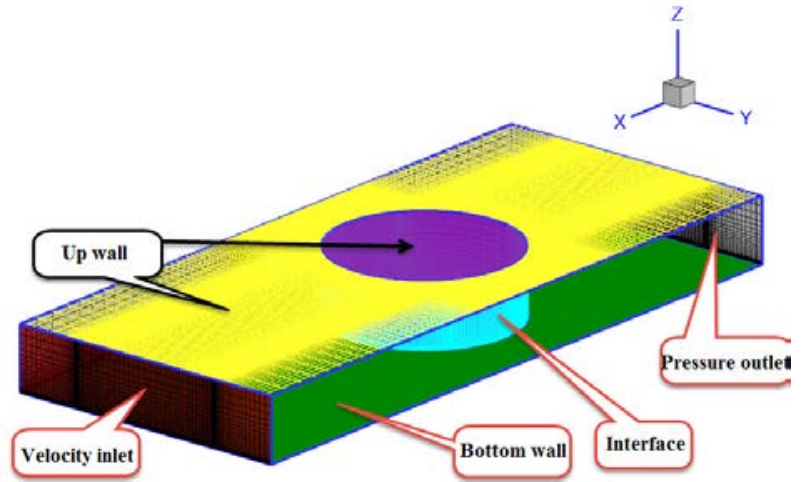

Fig.3 Boundary conditions

By employing the divided domains and the boundary conditions above, the variation of wind incidence angle is obtained, and flow parameters under different wall roughness conditions and different wind incidence angles, such as wind profiles, wind contour, etc., can be calculated.

\section{Automatic generation of mesh in inner domain}

Triangular surface mesh is first generated based on the geometry surface of micro-terrain, as shown in Fig.4. In order to guide air flow smoothly from plat ground to miro-terrain, smooth blending technique is used. Then prism mesh is generated based on surface triangular mesh. To avoid self-interaction of prism mesh elements, the technique proposed by David Eller ${ }^{[10]}$ is employed. Tetrahedral mesh is then generated by using Delaunay method based on the upper most triangular surface mesh of prism layers. The surface mesh on interface cylinder is triangular mesh for tetrahedron elements, while for prism layers, its surface mesh on interface cylinder is quadrilateral. The surface mesh of inner rotational domain is shown in Fig.5. 


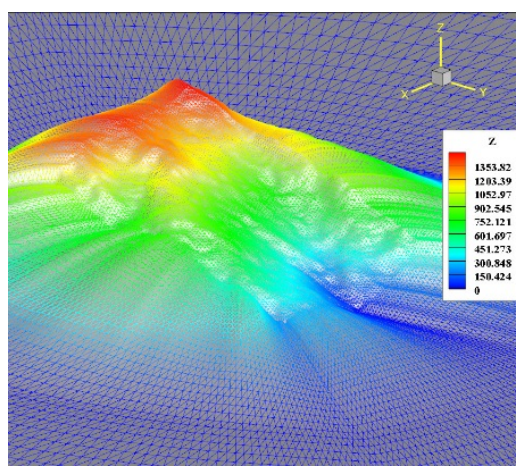

(a) perspective view

Figure 4 Triangular surface mesh

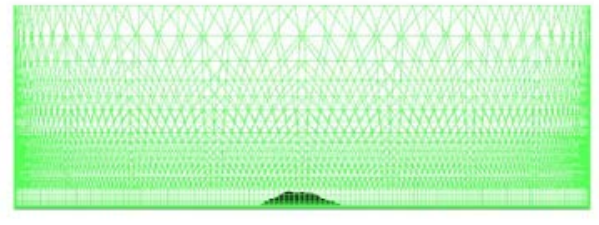

(b) interface and up-wall mesh

Figure 5 Mesh of inner domain

In order to view mesh element of interior mesh, the cut-view of prism/tetrahedron mesh at different $\mathrm{x}$ locations are plotted in Fig.6. From the figures below, it can be seen that, mesh size variation is smooth at the interface of prism layers and tetrahedron meshes.

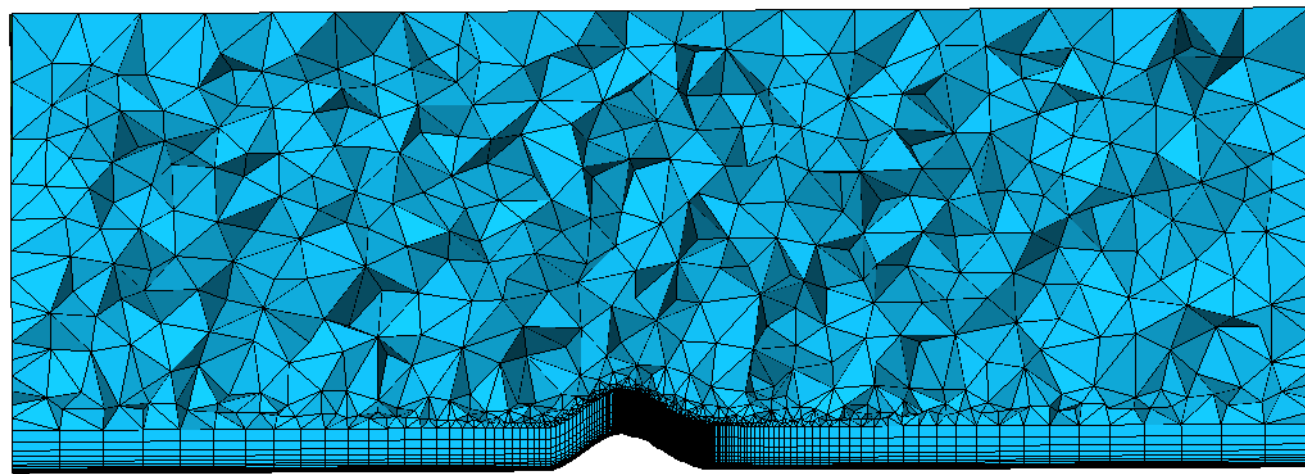

(a) cut-view of mesh at $x=0.0 \mathrm{~m}$ plane

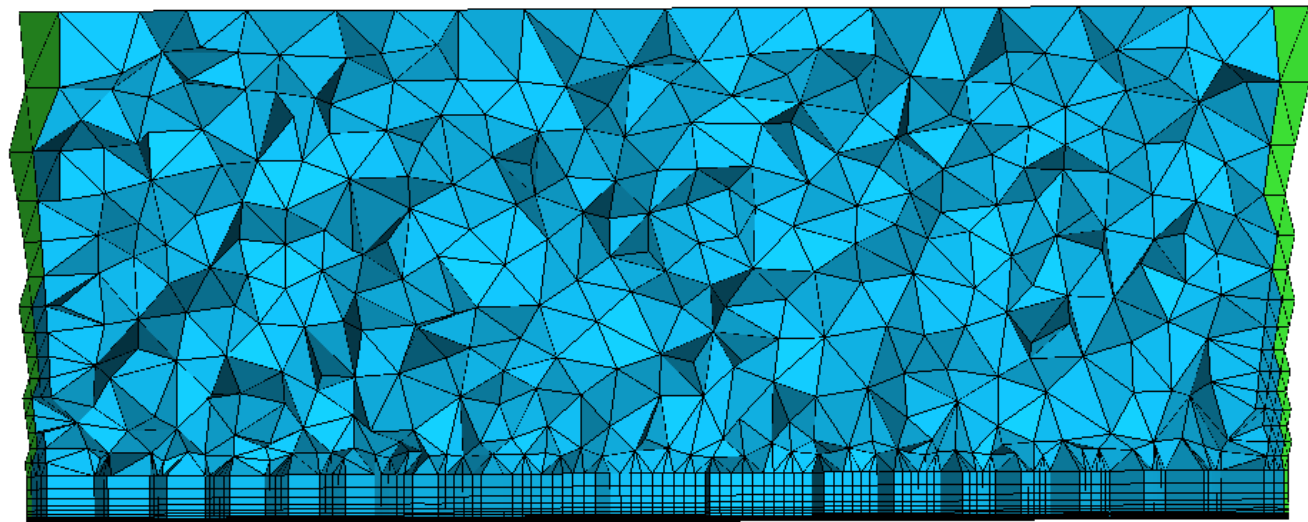

(b) cut-view of mesh at $\mathrm{x}=12500 \mathrm{~m}$ plane

Figure 6 Cut-view of mesh at different $\mathrm{X}$ planes

\section{Automatic generation of mesh in outer domain}

Given that the topology is very regular for outer zone, hence fully structured hexahedral mesh is generated. Especially, its height value is referred to that of interface cylinder of inner rotational domain. After mesh generation, the inner rotational domain and outer domain are assembled together, as shown in Fig.7. 


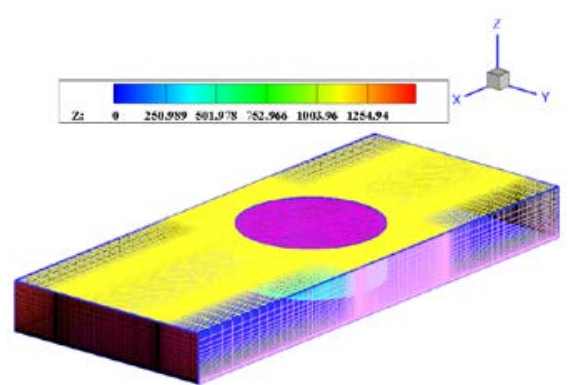

(a) isometric view

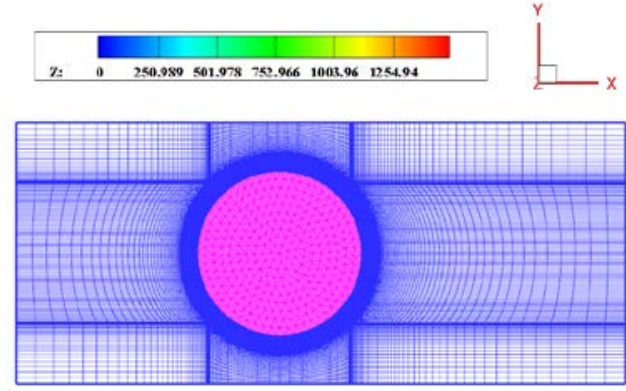

(b) top view

Fig.7 Assembled domains

\section{Mesh quality validation}

Accounting for the input data format of different commercial codes, the *.cas data format of fluent software is selected as the output file format. The generated mesh is then checked by using fluent software, and some of the checking results is presented as follows:

Reading "*.cas"...

Done.

Domain Extents:

x-coordinate: $\min (\mathrm{m})=-7.950000 \mathrm{e}+04, \max (\mathrm{m})=1.045000 \mathrm{e}+05$

y-coordinate: $\min (\mathrm{m})=-3.950000 \mathrm{e}+04, \max (\mathrm{m})=3.950000 \mathrm{e}+04$

z-coordinate: $\min (\mathrm{m})=0.000000 \mathrm{e}+00, \max (\mathrm{m})=1.764968 \mathrm{e}+04$

Volume statistics:

minimum volume (m3): $2.621650 \mathrm{e}+00$

maximum volume (m3): $3.147339 \mathrm{e}+10$

total volume (m3): $2.565198 \mathrm{e}+14$

Face area statistics:

Checking mesh

Done.

The checking results indicated that, there is no element with negative volume, thus this mesh can be used for CFD simulations.

\section{Conclusion}

In order to satisfy the wind resist demands of transmission lines crossing the hilly and mountainous zones, the automatic mesh generation technique for micro-terrains is investigated. The geometry surface of micro-terrain is obtained from ASTER GDEM database, and smooth blending technique is adopted to blend micro-terrain surface and plat ground surface. The computational domain is divided into inner rotational domain and outer domain to account for the influence of wind incidence angle; Delaunay triangulation is carried out for micro-terrain surface and then prism layers are generated, and then tetrahedron meshes are generated to fill the rest zone of inner domain; the mesh point height parameter is then copied and set to be the heights their corresponding outer zone mesh points, and fully hexagon mesh is generated for outer zone. Conservative flow data exchange is achieved by using interface boundary conditions inbetween inner and outer domains. The assembled domains are written in ".cas" data format of fluent commercial software, which is a general data format for unstructured mesh. The mesh is checked by using fluent software, and its quality satisfy the demands for CFD simulations. The whole process is carried out automatically by $\mathrm{C}++$ codes, thus not only human labour is greatly saved, but also analysis efficiency is improved. 


\section{Acknowledgements}

The research is funded by Technology Project of State Grid (High Resolution Wind Field Characteristics Study on Mountainous Terrain by Adaptive Simulation Technology).

\section{References}

[1] LU Qian. Study on Wind Turbine Distribution in Complex Geology[J]. Shanghai Power. 2008,(06):513-515.

[2] ZHOU Zhi-yong, XIAO Lian, DING Quan-sun, et al. Numerical Simulation Study of Wind Environment for the Flow around Large Region with Complex Terrain[J]. Chinese Quarterly of Mechanics, 2010,31(1):101-107.

[3] Daniel S. Abdi, Girma T. Bitsuamlak. Wind flow simulations on idealized and real complex terrain using various turbulence models[J]. Advances in Engineering Software, 75 (2014): 30-41.

[4] Qiang Du, DeshengWang. Recent progress in robust and quality Delaunay mesh generation[J]. Journal of Computational and Applied Mathematics, 195 (2006):8-23.

[5]J.D.Muller, P.L.Roe, H. Deconinck. A Frontal Approach for Node Generation in Delaunay Triangulations[J]. International Journal for numerical Methods in Engineering, 1993,17(3):241-255.

[6]P.J.Frey, Houman Borouchaki, Paul-Louis George. 3D Delaunay Mesh Generation Coupled with an Advancing Front Approach[J]. Computer Methods in Applied Mechanics \& Engineering, 1998, 157:115-131.

[7]WU Huo-zhen, JIAO Yu-yong, LI Hai-bo, et al. Study of 3D finite element tetrahedral mesh automatic generation for complex regions[J]. Rock and Soil Mechanics, 2011,32(11):3479-3486

[8]Jonathan Richard Shewchuk. Constrained Delaunay Tetrahedralizations and Provably Good Boundary Recovery[J]. Eleventh International Meshing Roundtable, 2002:193-204.

[9]Daming Feng, Christos Tsolakis, Andrey N. Chernikov. Scalable 3D Hybrid Parallel Delaunay Image-to-Mesh Conversion Algorithm for Distributed Shared Memory Architectures[J]. Procedia Engineering, 124 (2015):18-30.

[10]David Eller, Maximilian Tomac. Implementation and evaluation of automated tetrahedralprismatic mesh generation software[J]. Computer-Aided Design, http://dx.doi.org/10.1016;j.cad.2015.06.010. 